\title{
A near horizon extreme binary black hole geometry
}

\author{
Jacob Ciafre ${ }^{1, a}$, Maria J. Rodriguez ${ }^{1,2, b}$ \\ ${ }^{1}$ Department of Physics, Utah State University, 4415 Old Main Hill Road, Logan, UT 84322, USA \\ ${ }^{2}$ Max Planck for Gravitational Physics-Albert Einstein Institute, Am Muhlenberg 1, 14476 Potsdam, Germany
}

Received: 21 August 2018 / Accepted: 1 August 2019 / Published online: 11 September 2019

(C) The Author(s) 2019

\begin{abstract}
A new solution of four-dimensional vacuum General Relativity is presented. It describes the near horizon region of the extreme (maximally spinning) binary black hole system with two identical extreme Kerr black holes held in equilibrium by a massless strut. This is the first example of a non-supersymmetric, near horizon extreme binary black hole geometry of two uncharged black holes. The black holes are co-rotating, their relative distance is fixed, and the solution is uniquely specified by the mass. Asymptotically, the geometry corresponds to the near horizon extreme Kerr (NHEK) black hole. The binary extreme system has finite entropy.
\end{abstract}

\section{Contents}

1 Introduction ................. 1

2 Near-horizon extreme black hole binary geometry . 2

3 Discussion .................. 4

A Extreme co-rotating binary black hole review . . . . 5

References . . . . . . . . . . . . . . . 5 5

\section{Introduction}

Several black holes have been observed to rotate at nearly the speed of light [1-5]. Although there is considerable uncertainty in how black holes attain nearly extremal spins, several mechanisms in Nature can be envisioned. These include the merging of binary black holes (BBHs) possibly spinning at nearly extremal spins, and prolonged disk accretion. Numerical simulations, that are key in developing the most precise predictions of dynamical BBHs mergers [6-8] and black hole disk accretion [9-13], have confirmed the feasibility of these mechanisms giving rise to nearly extremal spinning black holes.

\footnotetext{
a e-mail: jakeciafre@outlook.com

be-mails: maria.rodriguez@aei.mpg.de; maria.rodriguez@usu.edu
}

From a theoretical perspective, it is conjectured that a rotating Kerr black hole obeys cosmic censorship: any singularities must lay behind the black hole's event horizon. As the black hole spin increases, for a fixed mass, the event horizon area protecting the singularity decreases. The maximum allowed spin is reached before exposing the singularity. These maximally rotating black holes are called extreme and are the central objects of study of this work.

At extremality, the black hole's temperature drops to zero. Interestingly, at this point the geometry close to the event horizon, the so called Near-Horizon Extreme Kerr (NHEK) geometry [14], displays a very special feature: the isometry group is enhanced to an $S L(2, R)$, conformal symmetry and develops a warped version of $A d S_{3}$. NHEK is obtained through scalings from the extreme Kerr black hole, has finite size, namely, finite event horizon area, and retains all the relevant aspects of black holes: a horizon and an ergosphere which may be associated with those corresponding to extreme Kerr. The enhanced conformal symmetry in NHEK, which does not extend to the full Kerr geometry, motivated works such as the Kerr/CFT conjecture concerning the quantum structure of black holes [15] and studies on the dynamics of stars [16] and energy extraction [17] in this region.

One then wonders, could NHEK be formed from a merger of the near horizon geometries of extreme BBHs? And, in this case, is the conformal symmetry also present in the geometry before merger giving rise to NHEK? To answer these questions, in this paper, we propose to focus on the stationary - rather than dynamical - BBHs and find explicitly the Near-Horizon Extreme Kerr Binary Black Hole geometry, "NHEK2". We will show that this new metric retains only partially the symmetry of NHEK, and are "tree"-like geometries: they are solutions with NHEK asymptotics, but as one moves inward the geometry branches into two smaller warped $A d S_{3}$ regions. Fragmentation of the $A d S$ near horizon extremal charged static black hole geometries was previously investigated in [18]. Further, a description in the dual CFT was presented where the $A d S$ trees in the geometry 
correspond to different classical vacua of a quantum mechanical theory.

Luckily, stationary (non-dynamical) BBHs solutions of Einstein's equations of General Relativity in vacuum with two-(neutral)Kerr black holes are also known analytically. These are exact asymptotically flat vacuum solutions with two spinning Kerr black holes supported by a conical singularity along the line separating the black holes. They represent special subfamilies of vacuum spacetimes constructed via a variety of solution-generating techniques in e.g. [1928]. The black holes in these solutions can co-rotate or spin in opposite directions. They become, for certain range of the parameters, extremal zero-temperature stationary BBHs solutions [25-28]. Remarkably, as observed in [22,26,27], the extreme BBHs overtake the extremal Kerr bound.

The fact that there is a conical singularity in all these solutions may seem discouraging, however the solution, and the contribution from the conical singularities have a well defined gravitational action [30]. Thus, standard Euclidean Gravity thermodynamical arguments can be employed, and one can extract new results from an apparently ill defined geometry. We interpret the conical singularities as boundary conditions necessary to prevent the collapse of the array and maintain the holes at fixed distance. . Note also that the total energy associated with the strut as seen by this observer at infinity is negative. For large separation $\Delta z$ respect to the mass of the black holes $M$, the energy is $E=-M^{2} G / \Delta z$, that corresponds to the Newtonian potential between two particles.

All of the above geometries were originally written in Weyl coordinates, where many expressions simplify. In these coordinates, the metric takes the form

$d s^{2}=-\frac{\hat{\rho}^{2}}{f} d \hat{t}^{2}+f(d \hat{\phi}+\omega d \hat{t})^{2}+e^{2 v}\left(d \hat{\rho}^{2}+d \hat{z}^{2}\right)$,

where $f, v, \omega$ are functions only of the $(\hat{\rho}, \hat{z})$-coordinates, and $\hat{t} \in(-\infty, \infty), \hat{\rho}, \hat{z} \in(-\infty, \infty)$ and $\hat{\phi} \sim \hat{\phi}+2 \pi$. Note that all stationary axis-symmetric black hole solutions can be written in Weyl coordinates (1), including the stationary BBHs central to our discussion, and also the Kerr and NHEK geometries. Our starting point to find the NHEK2 geometry are the co-rotating extreme double-Kerr black hole solutions in [28] (see Appendix A for a review). We develop an appropriate near-horizon limiting procedure in Weyl coordinates ${ }^{1}$, and apply it to the extreme BBHs geometry. As a result we obtain the new NHEK2 geometries, which becomes a distinct object with finite entropy. Our results may be of considerable interest in dual CFT studies as in $[15,18]$, entropy calculations, and gravitational signatures of near exremal spinning binary black holes.

1 The procedure to describe the near horizon geometries of extremal black holes has most frequently been developed in Boyer-Lindquist coordinates

\section{Near-horizon extreme black hole binary geometry}

The solution of extremal co-rotating identical BBHs [28] (that for completeness we review in Appendix A) from which we derive the new near-horizon geometry of extreme BBHs, takes a simpler representation in Weyl coordinates. We therefore perform the scaling computations in this frame. In this case, we find that the appropriate near-horizon limiting procedure for the extremal BBHs is

$\rho=\hat{\rho} \lambda, \quad z=\hat{z} \lambda$,

$t=\frac{\hat{t}}{\lambda}, \quad \phi=\hat{\phi}+\frac{\hat{t}}{2 M \lambda}$,

$p=-\frac{1}{\sqrt{2}}+\frac{(3 \sqrt{2}-2)}{4} \lambda \quad \kappa=M \lambda$,

taking $\lambda \rightarrow 0$ and keeping $(\hat{t}, \hat{\rho}, \hat{z}, \hat{\phi})$ fixed. In [28], $p$ and $\kappa$ are free parameters related to the definitions of positive mass and the separation between the black holes respectively. It is worth emphasizing that at the same rate of the zoom-in on the near-horizon region, the coordinate distance between the black holes is scaled to zero. The Newtonian distance between the black holes is $\Delta z=2 M$. Therefore, after the scaling procedure, the distance separating the black holes is finite, and yet the two black holes are held apart by a conical singularity (massless strut). As a result of this process, we find the near-horizon extreme black hole binary NHEK2 geometry in Weyl coordinates (1). This is defined by the equations

$$
\begin{aligned}
f & =-\frac{4 M^{2} \mu_{0}\left(\mu_{0}+2 \sigma_{0}^{2}\right)}{\mu_{0}\left(\mu_{0}+2 \sigma_{0}^{2}-2 \sigma_{1}+\pi_{0}\right)+\mu_{1} \pi_{1}+\left(1-y^{2}\right) \sigma_{0} \tau_{0}}, \\
\omega & =-\frac{\pi_{0} \sigma_{0}+\pi_{1} \sigma_{1}-\mu_{1}-4 \sigma_{0} \sigma_{1}-\left(1-y^{2}\right) \tau_{0} / 2}{2 M\left(\mu_{0}+2 \sigma_{0}^{2}\right)}, \\
e^{2 v} & =\frac{\mu_{0}\left(\mu_{0}+2 \sigma_{0}^{2}-2 \sigma_{1}+\pi_{0}\right)+\mu_{1} \pi_{1}+\left(1-y^{2}\right) \sigma_{0} \tau_{0}}{K_{0}^{2}\left(x^{2}-y^{2}\right)^{4}},
\end{aligned}
$$

where the functions yield

$$
\begin{aligned}
\mu_{0}= & -\frac{\hat{\rho}^{2}}{2 M^{2}}, \quad \sigma_{0}=-\frac{x^{2}-y^{2}}{2}-\frac{x^{2}+y^{2}}{\sqrt{2}}, \\
\pi_{1}= & -2 \sqrt{2} x^{2}-(1-\sqrt{2})\left(x^{2}-y^{2}\right) \\
\mu_{1}= & -\frac{(3-\sqrt{2})}{2}\left(-1+x^{2}\right)^{2}+(1-\sqrt{2})\left(x^{2}-y^{2}\right)^{2} \\
\sigma_{1}= & \frac{(3-\sqrt{2})}{2}\left(x^{2}-y^{2}\right) \\
& +\frac{(1-\sqrt{2})(3-2 \sqrt{2})}{2 \sqrt{2}}\left(x^{2}+y^{2}\right) \\
\pi_{0}= & \left(\frac{-74+85 \sqrt{2}}{14}\right) x^{2}-\sqrt{2} x\left(1+x^{2}\right) \\
& +\left(\left(\frac{145-108 \sqrt{2}}{28}\right)-x\right)\left(x^{2}-y^{2}\right)
\end{aligned}
$$




$$
\begin{aligned}
\tau_{0}= & \sqrt{2} x\left(x^{2}-1\right)-\left(\frac{99-51 \sqrt{2}}{28}-(1-\sqrt{2}) x\right) \\
& \left(x^{2}-y^{2}\right)+\left(\frac{71-23 \sqrt{2}}{28}\right)\left(1-y^{2}\right),
\end{aligned}
$$

and

$x=\frac{\sqrt{\hat{\rho}^{2}+(\hat{z}+M)^{2}}+\sqrt{\hat{\rho}^{2}+(\hat{z}-M)^{2}}}{2 M}$,

$y=\frac{\sqrt{\hat{\rho}^{2}+(\hat{z}+M)^{2}}-\sqrt{\hat{\rho}^{2}+(\hat{z}-M)^{2}}}{2 M}$,

with

$K_{0}=-(1+\sqrt{2}) / 2$.

We have checked that this metric is Ricci flat and, thus, a solution to the vacuum Einstein equations except on the axis between the two black holes where the strut is placed, and this has an effective stress-energy. This solution represents the near horizon geometry of two extreme Kerr black holes. With this procedure, the asymptotically flat Minkowski region from the original metric decouples and the throat becomes infinitely long while there is a splitting into two pieces that survives.

Horizons. Our solution displays two horizons located at

$\hat{\rho}_{H}=0, \quad \hat{z}_{H}= \pm M$.

Besides the conical singularity (to be analyzed in more detail below) the metric is smooth. The NHEK2 geometry is not asymptotically flat; in fact, as we show in more detail below, it approaches the NHEK asymptotic geometry..

There are two $U(1)$ symmetries $-\partial_{\hat{t}}$ and $\partial_{\hat{\phi}}$ symmetries - present in the NHEK2 metric; the metric lacks one of the killing vectors that would otherwise close in $S L(2, R)$ as in NHEK. An enhancement of the isometry to $S L(2, R) \times U(1)$ is observed only when the two black holes collapse into one, and form a single larger black hole. In the collapse of NHEK2 to NHEK, the distance between the black holes goes to zero and corresponds to the asymptotic limit of NHEK2 as we describe here below.

Inspection of the NHEK2 solution shows that changing coordinates as

$$
\begin{aligned}
\hat{t} \rightarrow & (-2+\sqrt{2}) M^{2} T, \quad \hat{\rho} \rightarrow(R \pm M) \sin \Theta, \\
\hat{z} \rightarrow & (R \pm M) \cos \Theta \mp M, \\
& \phi \rightarrow(-2+\sqrt{2}) T+\frac{(148-107 \sqrt{2})}{56} M \Phi,
\end{aligned}
$$

the expansions close to each of the black holes - located now at $R= \pm M$ - gives rise to a metric of the form

$$
\begin{gathered}
d s^{2} \sim M^{2} \Gamma(\Theta)\left[-(R \pm M)^{2} d T^{2}+\frac{d R^{2}}{(R \pm M)^{2}}\right. \\
\left.+d \Theta^{2}+\Lambda(\Theta)(d \Phi+(R \pm M) d T)^{2}\right]
\end{gathered}
$$

with

$$
\begin{aligned}
\Gamma(\Theta) & =\frac{((22-15 \sqrt{2})(3+\cos 2 \Theta) \pm(72-52 \sqrt{2}) \cos \Theta)}{8}, \\
\Gamma(\Theta) \Lambda(\Theta) & =\frac{16(2-\sqrt{2})^{2} \sin ^{2} \Theta}{((6-\sqrt{2})(3+\cos 2 \Theta) \pm(8-12 \sqrt{2} \cos \Theta))},
\end{aligned}
$$

when the leading terms in each metric component are retained. Hence locally, in the vicinity of each black hole, we note that the slices of the geometry at fixed polar angle $\Theta$ correspond to warped $A d S_{3}$. Our newly discovered metric are "tree"-like geometries: they are solutions with NHEK asymptotics, but as one moves inward the geometry branches into two smaller warped $A d S_{3}$ regions. For the same special constant value of $\Theta=\Theta_{0}$ where $\Lambda\left(\Theta_{0}\right)=1$, the local metrics are that of $A d S_{3}$. Other tree-like geometries have been previously found for extreme charged BBHs [18]. Whether this local manifestation of two copies of $A d S_{3}$ in NHEK hints really to an underlying conformal symmetry of the extreme BBHs is yet a question that needs further investigation.

Following [22,29], the horizon area can be computed from the metric (15) close to each of the black holes via

$A_{i} \equiv 2 \pi \int_{0}^{\pi} \sqrt{g_{\Theta \Theta} g_{\Phi \Phi}} d \Theta$.

The corresponding entropy $S_{i}=A_{i} / 4, i=1,2$ of the extreme constituents is

$S_{1}=S_{2}=(2-\sqrt{2}) \pi M^{2} \approx 0.5858 \times \pi M^{2}$.

The total entropy of the system is

$S_{N E H K 2}=S_{1}+S_{2}=(2-\sqrt{2}) 2 \pi M^{2}$.

Asymptotically, for $\hat{\rho}=r \sin \theta, \hat{z}=r \cos \theta$ and $r \rightarrow \infty$, the limiting metric is also finite but no longer flat. In the asymptotic limit, it corresponds to the NHEK metric - in Weyl coordinates - (1) with functions

$$
\begin{aligned}
f & =\frac{4 M^{2} \hat{\rho}^{2}}{2 \hat{z}^{2}+\hat{\rho}^{2}}, \quad \omega=\frac{\sqrt{\hat{z}^{2}+\hat{\rho}^{2}}}{2 M^{2}}, \\
e^{2 v} & =\frac{M^{2}\left(2 \hat{z}^{2}+\hat{\rho}^{2}\right)}{\left(\hat{z}^{2}+\hat{\rho}^{2}\right)^{2}} .
\end{aligned}
$$

The black holes in the NHEK2 solution (1) with functions (5)-(13) collapse into one. The collapse geometry corresponds to the single NHEK black hole metric. Each of the black holes in the original NHEK2 geometry contributes a mass $M / 2$. The larger NHEK black hole formed after the collapse has mass $M_{N H E K}=M$. The entropy in this case is

$S_{N H E K}=2 \pi M^{2}$.

A more detailed discussion about the entropy of extremal binary black holes (18). 
Conical singularity. We now compute the conical singularities of the new NHEK2 metric

$\Delta \hat{\phi}=2 \pi \lim _{\hat{\rho} \rightarrow 0}\left(1-\sqrt{\frac{f}{\hat{\rho}^{2} e^{2 v}}}\right), \quad-M<z<M$,

While the NHEK2 metric has no naked curvature singularities, our computation show that there is a non-removable conical deficit between the two horizons

$\Delta \hat{\phi}=2 \pi(1 / 2+\sqrt{2})$.

Charges and entropy. The mass and angular momenta of the solution follow from the limiting near-horizon procedure of the extreme BBHs solution that is manifestly asymptotically flat

$M_{1}=M_{2}=M / 2, \quad J_{1}=J_{2}=M^{2} / 2$,

Interestingly, the ratio $J_{1} / M_{1}^{2}=J_{2} / M_{2}^{2}=2$ is fixed. While Hawking's temperature is zero for NHEK2, each black hole has an angular velocity

$\Omega_{1}=\Omega_{2}=1 /(2 M)$.

It is straightforward to verify that the Smarr law (for the extremal, zero temperature configuration) is satisfied $M_{i}=$ $2 \Omega_{i} J_{i}$ for each individual black hole $i=1,2$.

Ergosphere. There are regions in the NHEK2 spacetime where the vector $\partial_{\hat{t}}$ becomes null. We will refer to the boundary region as the ergosphere, since they appear as a consequence of the presence of such regions in the original stationary extreme BBHs geometries. For NHEK2 these are defined by regions where $g^{\hat{t} \hat{t}}=0$ and give rise to a set of disconnected regions as shown in Fig. 1. The horizons of the black holes in NHEK 2 are points in the $(\hat{\rho}, \hat{z})$-plane and have finite horizon areas. There is a self similar behavior close to each black hole that resembles the ergospheres of isolated extremal Kerr black hole.

Uniqueness. The NHEK2 geometry that we constructed is asymptotically NHEK, and contains two, instead of one, horizons in the bulk held in equilibrium by a massless strut. It represents the first example of a geometry with NHEK asymptotics that is not diffeomorphic to NHEK, which hence explicitly shows the non-uniqueness of metrics with NHEK asymptotics with non-smooth horizon. This is not in contradiction with the findings of $[38,39]$, where only asymptotically NHEK solutions with smooth horizons are considered. Moreover, for a fixed value of the total mass, it is expected that other finite near-horizon extremal BBHs with localized conical singularities (or equivalently non-smooth horizons) exist. These may include extremal BBHs containing two black holes with non-identical masses or with spins that are not aligned. Another intriguing aspects related to the uniqueness includes the classifications of near horizon geometries

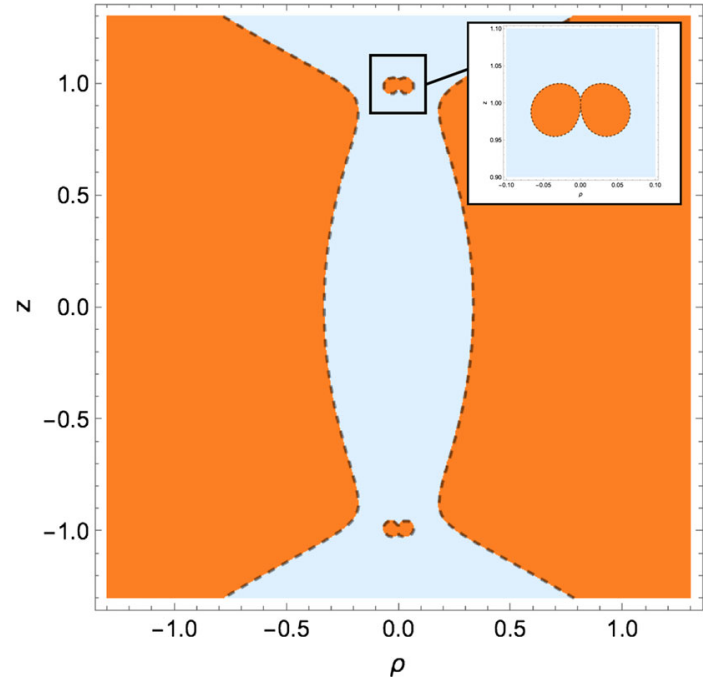

Fig. 1 Ergoregion (shaded orange region) of the NHEK2 black hole solution for $M=1$, as a consequence of the presence of such regions in the original stationary extreme $\mathrm{BBH}$ geometries. The black holes are located at $\rho=\hat{\rho}_{H}=0, z=\hat{z}_{H}= \pm 1$. A more detailed diagram, close to one of the black holes appears in the upper right corner. The dashed line corresponds to the boundary where $\partial_{\hat{t}}$ is null

in [31,32]. NHEK2 does not pertain to any of the classes considered therein. This suggests that a new set of assumptions are needed for a complete classification i.e. restricting the number of isolated horizons in the solutions.

\section{Discussion}

The extremal BBHs solutions [28], as well as the new NHEK2 geometries that we have found in this note, contain a localized conical singularity (strut), in the line separating the two black holes. Conical singularities are, in contrast to curvature singularities in the geometry, possibly avoidable as an orbital spin is reinstated. In the stationary BBHs that we have focused on, for example, one could introduce a rotation along one of the planes perpendicular to the azimuthal-axis. This mechanism would give rise to a centrifugal force, that may balance the self-gravitational effects between the two black holes and eliminate the strut. So far, however, this modification of the stationary BBHs solutions has not been achieved analytically. Other solutions where rotation plays a role in balancing different configurations have been given in [33-35]. ${ }^{2}$ Another reason for considering the stationary rather than the dynamical BBHs solutions is their extremal maximally rotating (neutral) counterparts.

\footnotetext{
2 The possibility of adding a rotation to the BBH system to cancel the strut may be achieved by breaking the axisymmetry while preserving stationarity, or perhaps via a more challenging approach by solving the dynamical problem perturbatively.
} 
Progress has been reported on rapidly spinning dynamical BBHs mergers [6-8], but extremal zero temperature BBHs are not viable yet through numerical explorations. Therefore, to study the extremal BBHs one would have to choose to work with the exact extreme stationary BBHs and consider the effect of the conical singularities in these solutions. While conical singularities are not desirable, these have been shown to be irrelevant to the so called black hole shadows [36,37] thanks to the underlying cylindrical symmetry of the problem. This suggests that certain physical properties of black holes are protected by symmetries and can be studied even with solutions that contain localized conical singularities.

Acknowledgements The authors would like to thank P. Cunha, S. Hadar and C. Herdeiro for helpful discussions and O. Varela for careful reading of the manuscript. This work was supported by the NSF Grant PHY-1707571 at Utah State University and the Max Planck Gesellschaft through the Gravitation and Black Hole Theory Independent Research Group.

Data Availability Statement This manuscript has no associated data or the data will not be deposited. [Authors' comment: This work is involves only analytical results and no data is generated.]

Open Access This article is distributed under the terms of the Creative Commons Attribution 4.0 International License (http://creativecomm ons.org/licenses/by/4.0/), which permits unrestricted use, distribution, and reproduction in any medium, provided you give appropriate credit to the original author(s) and the source, provide a link to the Creative Commons license, and indicate if changes were made.

Funded by SCOAP S $^{3}$.

\section{A Extreme co-rotating binary black hole review}

For completeness in this Appendix we review the exact solution of four-dimensional vacuum General Relativity corresponding to two extremal co-rotating Kerr BHs with a localized strut between the BHs as it was identified beautifully in [28]. This is an asymptotically flat metric of the form (1)

$d s^{2}=-\frac{\rho^{2}}{f} d t^{2}+f(d \phi+\omega d t)^{2}+e^{2 v}\left(d \rho^{2}+d z^{2}\right)$

with metric functions defined by

$$
\begin{aligned}
& f=\frac{\kappa\left(y^{2}-1\right) F}{D \omega}, \quad e^{2 v}=\frac{D}{K_{0}^{2}\left(x^{2}-y^{2}\right)^{4}}, \\
& \omega=-\frac{\kappa\left(y^{2}-1\right) F N}{\left[\left(\kappa\left(y^{2}-1\right) F\right)^{2}-\rho^{2} D^{2}\right]},
\end{aligned}
$$

where

$$
\begin{aligned}
& x=\frac{\sqrt{\rho^{2}+(z+\kappa)^{2}}+\sqrt{\rho^{2}+(z-\kappa)^{2}}}{2 \kappa}, \\
& y=\frac{\sqrt{\rho^{2}+(z+\kappa)^{2}}-\sqrt{\rho^{2}+(z-\kappa)^{2}}}{2 \kappa},
\end{aligned}
$$

and

$$
\begin{aligned}
N= & \mu^{2}-\left(x^{2}-1\right)\left(1-y^{2}\right) \sigma^{2}, \\
D= & N+\mu \pi+\left(1-y^{2}\right) \sigma \tau, \\
F= & \left(x^{2}-1\right) \sigma \pi+\mu \tau, \\
\mu= & p^{2}\left(x^{2}-1\right)^{2}+q^{2}\left(1-y^{2}\right)^{2}+\left(\alpha^{2}-\beta^{2}\right)\left(x^{2}-y^{2}\right)^{2}, \\
\sigma= & 2\left[p q\left(x^{2}-y^{2}\right)+\beta\left(x^{2}+y^{2}\right)-2 \alpha x y\right], \\
\pi= & \frac{4 p^{2}}{K_{0}}\left\{K_{0}\left[p x\left(x^{2}+1\right)+2 x^{2}\right]\right. \\
& \left.+\left(x^{2}-y^{2}\right)\left(K_{0} q \beta x-2 p^{4} \beta^{2}\right)+4 p^{2}(p q+\beta) \beta x^{2}\right\}, \\
\tau= & \frac{4 p^{2}}{K_{0}}\left\{K_{0} x\left[q\left(x^{2}-1\right)-(q-p \beta)\left(x^{2}-y^{2}\right)\right]\right. \\
& \left.+2 p^{3}(p+q \beta) \beta+p^{2}(p q+\beta)\left(y^{2}-1\right)\right\},
\end{aligned}
$$

and constants

$$
\begin{aligned}
& K_{0}=p^{2}\left(p^{2}-\beta^{2}\right), \quad \beta=\frac{1}{2 p}\left[\Delta_{\mathrm{S}}+q(1+p)\right], \\
& \Delta_{\mathrm{S}}=\left[(1+p)\left(1+3 p^{2}+p q^{2}\right)\right]^{1 / 2}, \quad q^{2}=1-p^{2} .
\end{aligned}
$$

The positive values of the mass correspond to the parameter range

$$
-\frac{1}{\sqrt{2}} \leq p<0
$$

In this coordinates the event horizons of the extremal black holes are two points located at

$\rho_{H}=0, \quad z_{H}= \pm \kappa$.

\section{References}

1. L. Gou et al., The extreme spin of the black hole in cygnus X-1. Astrophys. J. 742, 85 (2011). arXiv:1106.3690 [astro-ph.HE]

2. K.A. Pounds, J.N. Reeves, A.R. King, K.L. Page, Exploring the complex X-ray spectrum of NGC 4051. Mon. Not. R. Astron. Soc. 350, 10 (2004). arXiv:astro-ph/0310257

3. G. Risaliti et al., A rapidly spinning supermassive black hole at the centre of NGC 1365. Nature 494, 449 (2013). arXiv: 1302.7002 [astro-ph.HE]

4. A.C. Fabian, The innermost extremes of black hole accretion. Astron. Nachr. 337(4/5), 375 (2017). arXiv:1511.08596 [astroph.HE]

5. J.E. McClintock, R. Shafee, R. Narayan, R.A. Remillard, S.W. Davis, L.X. Li, The spin of the near-extreme Kerr black hole GRS 1915+105. Astrophys. J. 652, 518 (2006). arXiv:astro-ph/0606076

6. L. Rezzolla, E.N. Dorband, C. Reisswig, P. Diener, D. Pollney, E. Schnetter, B. Szilagyi, Spin diagrams for equal-mass blackhole binaries with aligned spins. Astrophys. J. 679, 1422 (2008). arXiv:0708.3999 [gr-qc]

7. M. Kesden, G. Lockhart, E.S. Phinney, Maximum black-hole spin from quasi-circular binary mergers. Phys. Rev. D 82, 124045 (2010). arXiv:1005.0627 [gr-qc]

8. G. Lovelace, M.A. Scheel, B. Szilagyi, Simulating merging binary black holes with nearly extremal spins. Phys. Rev. D 83, 024010 (2011). arXiv:1010.2777 [gr-qc] 
9. M. Volonteri, P. Madau, E. Quataert, M.J. Rees, The distribution and cosmic evolution of massive black hole spins. Astrophys. J. 620, 69 (2005). arXiv:astro-ph/0410342

10. E. Berti, M. Volonteri, Cosmological black hole spin evolution by mergers and accretion. Astrophys. J. 684, 822 (2008). arXiv:0802.0025 [astro-ph]

11. K.S. Thorne, Astrophys. J. 191, 507 (1974)

12. C.F. Gammie, S.L. Shapiro, J.C. McKinney, Black hole spin evolution. Astrophys. J. 602, 312 (2004). arXiv:astro-ph/0310886

13. S.L. Shapiro, Spin, accretion and the cosmological growth of supermassive black holes. Astrophys. J. 620, 59 (2005). arXiv:astro-ph/0411156

14. J.M. Bardeen, G.T. Horowitz, The extreme Kerr throat geometry: a vacuum analog of $\operatorname{AdS}(2)$ x S**2. Phys. Rev. D 60, 104030 (1999). arXiv:hep-th/9905099

15. M. Guica, T. Hartman, W. Song, A. Strominger, The Kerr/CFT correspondence. Phys. Rev. D 80, 124008 (2009). arXiv:0809.4266 [hep-th]

16. S. Hadar, A.P. Porfyriadis, A. Strominger, Gravity waves from extreme-mass-ratio plunges into Kerr black holes. Phys. Rev. D 90(6), 064045 (2014). arXiv:1403.2797 [hep-th]

17. A. Lupsasca, M.J. Rodriguez, A. Strominger, Force-free electrodynamics around extreme Kerr black holes. JHEP 1412, 185 (2014). arXiv: 1406.4133 [hep-th]

18. J.M. Maldacena, J. Michelson, A. Strominger, Anti-de Sitter fragmentation. JHEP 9902, 011 (1999). https://doi.org/10.1088/ 1126-6708/1999/02/011. arXiv:hep-th/9812073

19. W. Kinnersley, D.M. Chitre, Symmetries of the stationary EinsteinMaxwell equations. IV. Transformations which preserve asymptotic flatness. J. Math. Phys. 19, 2037 (1978)

20. V.S. Manko, E. Ruiz, Extended multi-soliton solutions of the Einstein field equations. Class. Quantum Gravity 15, 2007 (1998)

21. V.S. Manko, E.D. Rodchenko, E. Ruiz, B.I. Sadovnikov, On the simplest binary system of rotating black holes. AIP Conf. Proc. 1122, 332 (2009)

22. C.A.R. Herdeiro, C. Rebelo, On the interaction between two Kerr black holes. JHEP 0810, 017 (2008). arXiv:0808.3941 [gr-qc]

23. V.S. Manko, E.D. Rodchenko, E. Ruiz, B.I. Sadovnikov, Exact solutions for a system of two counter-rotating black holes. Phys. Rev. D 78, 124014 (2008). arXiv:0809.2422 [gr-qc]
24. V.A. Belinski, V.E. Zakharov, Stationary gravitational solitons with axial symmetry. Sov. Phys. JETP 50(1), 1 (1979)

25. D. Kramer, G. Neugebauer, The superposition of two Kerr solutions. Phys. Lett. A. 75(4), 259 (1980)

26. V.S. Manko, J. Martin, E. Ruiz, On the simplest binary system of stationary black holes. Phys. Lett. A. 196, 23 (1994)

27. M.S. Costa, C.A.R. Herdeiro, C. Rebelo, Dynamical and thermodynamical aspects of interacting Kerr Black holes. Phys. Rev. D 79, 123508 (2009). arXiv:0903.0264 [gr-qc]

28. V.S. Manko, E. Ruiz, On a simple representation of the Kinnersley-Chitre metric. Prog. Theor. Phys. 125, 1241 (2011). arXiv: 1101.4687 [gr-qc]

29. M.S. Costa, M.J. Perry, Interacting black holes. Nucl. Phys. B 591, 469 (2000). arXiv:hep-th/0008106

30. G.W. Gibbons, M.J. Perry, New gravitational instantons and their interactions. Phys. Rev. D 22, 313 (1980). arXiv:1101.4687 [gr-qc]

31. H.K. Kunduri, J. Lucietti, H.S. Reall, Near-horizon symmetries of extremal black holes. Class. Quantum Gravity 24, 4169 (2007). arXiv:0705.4214 [hep-th]

32. H.K. Kunduri, J. Lucietti, Classification of near-horizon geometries of extremal black holes. Living Rev. Relativ. 16, 8 (2013). arXiv:1306.2517 [hep-th]

33. R. Emparan, H.S. Reall, Generalized Weyl solutions. Phys. Rev. D 65, 084025 (2002). arXiv:hep-th/0110258

34. R. Emparan, D. Mateos, P.K. Townsend, Supergravity supertubes. JHEP 0107, 011 (2001). arXiv:hep-th/0106012

35. O. Lunin, S.D. Mathur, Metric of the multiply wound rotating string. Nucl. Phys. B 610, 49 (2001). arXiv:hep-th/0105136

36. P.V.P. Cunha, C.A.R. Herdeiro, M.J. Rodriguez, Does the black hole shadow probe the event horizon geometry? arXiv:1802.02675 [gr-qc]

37. P.V.P. Cunha, C.A.R. Herdeiro, M.J. Rodriguez, Shadows of exact binary black holes. Phys. Rev. D 98(4), 044053 (2018)

38. A.J. Amsel, G.T. Horowitz, D. Marolf, M.M. Roberts, No dynamics in the extremal Kerr throat. JHEP 0909, 044 (2009). arXiv:0906.2376 [hep-th]

39. O.J.C. Dias, H.S. Reall, J.E. Santos, Kerr-CFT and gravitational perturbations. JHEP 0908, 101 (2009). arXiv:0906.2380 [hep-th] 